\title{
Vacuum-caps membranes' equilibrium state forms based on the energy criterion
}

\section{Oleksandr Vatrenko', Volodymyr Kyrylov' ${ }^{1}$, Oleksandr Gavva ${ }^{2}$}

1 - Odesa National Academy of Food Technologies, Odesa, Ukraine.

2 - National University of Food Technolog, Kyiv, Ukraine.

Keywords:

Vacuum-cap

Membrane

Equilibrium

Deflection

Pressure

\section{Article history:}

Received

24.06.2019

Received in

revised form

01.10 .2019

Accepted

30.03 .2020

\section{Corresponding}

author:

\section{Oleksandr}

Vatrenko

E-mail:

alexvatrenko@

gmail.com

\section{DOI:}

$10.24263 / 2304-$

974X-2020-9-1-

16

\section{Abstract}

Introduction. In order to describe the energy transformation that conditions the membranes' stable operation under a given mode of product processing, the studies of glass containers' vacuum caps membranes equilibrium state forms have been carried out.

Materials and methods. The glassware containers represent the study object hereunder, the research subject being their caps' flexible membranes. Instrumental methods have been used to assess the membrane thickness and deflection. The forms of equilibrium state and membrane equilibrium energy levels were investigated through mathematical simulation by energy modeling.

Results obtained and discussion. After loss of stability, the membrane shifts into a different position of stable equilibrium. The measurement results showed that at stability loss state, the membrane center additional deflection is $f=0,07 \mathrm{~mm}$, that is significantly less than the membrane center initial deflection value, $f_{\text {init }}=0,25 \mathrm{~mm}$. Therefore, as a result of the stability loss the membrane working cone is not subjected to the mirror deformation. These deflection parameters ensure that the membranes are operable over a wide range of drop between the system backpressure (at an autoclave) and the container internal pressure throughout the product thermal processing.

The circular plate total energy is represented as the sum of the zerotorque stress state energy, bending energy and the external pressure work values. Calculated is the derived membrane total energy equation, obtained are the membrane energy levels for different stable equilibrium states.

Substituting the experimentally obtained value $f$ in the calculated equation of stability loss state membrane total energy we get the cap membranes stability loss pressure value calculated for the considered case, $P_{1}=0,0326 \cdot 10^{6} \mathrm{~Pa}$. The calculated value $P_{l}$ is close to the cap membranes stability loss pressure value $P_{l}=0,03 \mathrm{MPa}$, specified by the caps manufacturer. The $P_{I}$ pressure calculated value deviation from the real one can be influenced by the hardness of tin used to produce the cap bearing such membrane.

The obtained energy levels of membrane's different equilibrium states at two different loads do correspond to the critical pressures of stability loss $\mathrm{P}_{1}{ }^{*}$ and load releasing $\mathrm{P}_{2}{ }^{*}$. Both pressures energy levels diagrams built using the calculated equation, include the potential energy minimums. This form of the energy criterion curves for critical pressures is completely consistent with the Lagrange-Dirichlet theorem, since the pressure $\mathrm{P}_{1 \mathrm{~min}}{ }^{*}$ corresponds to the energy level minimum relative to other adjacent states.

Conclusions The membrane total energy score equation and the energy levels for the equilibrium states corresponding to the critical pressures have been obtained. 


\section{Introduction}

Modern capping elements for glass containers of various capping systems contain membranes specific with a small initial deflection and, depending on their positively or negatively convex state indicate the vacuum presence or absence in the packed container, and, consequently, its tightness [1].

Depending on the glass container size and the mode of heat treatment, the caps are made of different thickness and hardness tin, i.e. tin of different energy levels [2]. In modern science, there are several methods for calculating thin plates. They are presented in $[3,4,5]$. The authors calculate mainly thin flat plates under different types of load. However, thin plates with an initial deflection due to the load applied, making this study subject, have not been considered in those sources.

The thin plates calculation energy method exposed by numerous authors, is widely known and effectively used [6, 7, 8]. The article [9] uses the energy method to solve the local bending problem for a thin-walled cylindrical steel tube with elastic filling under the concentric axial load applied. The problems of calculating thin plates that bear an initial deflection to counteract the load are not reflected in those works.

The study of Volmir A. [10] describes the operation of initially deflected round flexible plates, fixed in various ways, at different, relative to the initial deflection, directions of applied load action. However, it is presented in general form and is not supported with a complete scientific calculation that would describe and explain the membranes operation including the consideration of basic material energy component.

In the study by Timoshenko S. et al. [11], approximate formulas for calculating an uniformly loaded round plate with significant deflections are given. In [12], the general Lagrangian formulation for flexible plates is considered accordingly to the finite element method for plates under large displacement and rotation applied. The round plates with small displacement studied in our research were not considered therein.

Regarding the metal packaging containers, the vacuum cap membranes operation studies have been carried out. In [1], an equation for the relationship between pressure, geometric parameters, and membrane thickness has been obtained. In [13], described is a simulation as to the deformation behavior of the canned glass containers metal caps membranes during storage and processing of packaged products. On the basis of the obtained scope equation, a computer program has been developed for building the "pressure deflection" relationship model. Obtained are the membranes deformation characteristics depending on changes in the tin sheet initial deflection and thickness [13]. However, the membrane total energy change during the deformation process, and primarily in the equilibrium states, has not been studied thus implying such study necessity. As a result, certain difficulties arise regarding the elastic plate functional reliability and its design.

This study is purposed to describe the initially deflected round plate's energy change that causes the membranes' stable operation in a given mode.

This study objectives:

1. To solve the equations of local energy effects on the membrane during its operation and to get the membrane system total energy scope equation.

2. To assess experimentally the membrane additional deflection in the working position.

3. To check the obtained equation adequacy for the operation of glass containers metal caps real membranes.

4. To build graphs of the membrane energy levels according to its characteristic equilibrium states. 


\section{Materials and methods}

\section{Studied materials}

For experimental studies, we used caps with a "safety button" according to the "twistoff " system [2] with a diameter of $82 \mathrm{~mm}$, produced by the German company Silgan White cap, and used by the EU and Ukrainian canning enterprises.

Structurally, the membranes are designed to operate in a controlled stability loss mode, Figure 1a. The membranes include a support cone of $D_{3}$ outer diameter, a working cone of $D_{2}$ outer diameter, and a flat section of $D_{1}$ diameter. They lose their stability due to the vacuum occurring in the container that causes a pressure drop on the cap field. Further we refer to this pressure drop as to the pressure (applied).

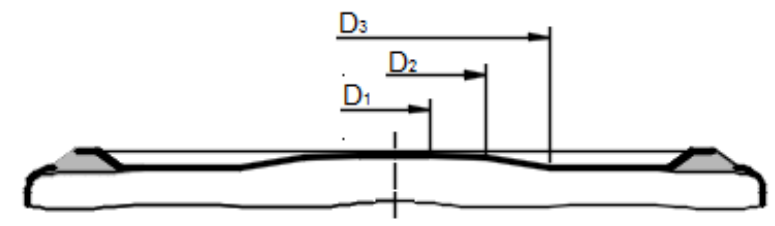

a)

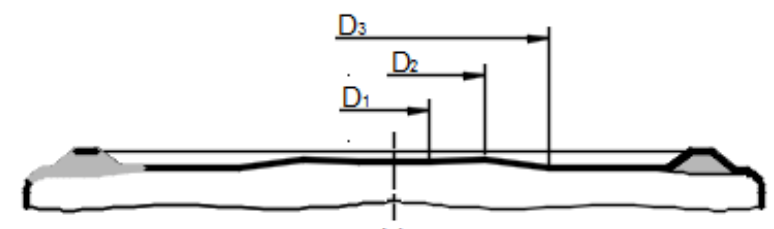

b)

Figure 1. Cap field:

a - membrane non-loaded state; $b$ - membrane stability loss state.

Next to the stability loss, the membrane moves to the stable equilibrium state, Figure 1b. The values of stability loss and shape restoring critical pressure which determine the existing membranes operation interval are generally known and some companiesmanufacturers of glassware caps do specify those values

Accordingly, this research object is a glass packaging container with vacuum cap. The research subject represents the flexible membranes of metal caps.

The membrane center additional deflection in the stability loss state was determined experimentally.

\section{Preparing the experimental samples}

The packaging samples have been prepared in production enterprise conditions. The caps were fed to a steam-vacuum capping machine, where they were used to seal glass jars with products in the operating mode. Then the product was cooled reaching the ambient temperature to create a vacuum in the empty container volume, after that the tested sample was ready for experiment. 


\section{Procedure for research conducting}

The round plate potential energy depends on the geometry of its cross-section (configuration). In the analytical study of the membrane equilibrium state kinds we will adhere to the design scheme, when the membrane working cone is pinched along the contour with a free radial displacement of the contour points, since it is closest to reality (Figure 2). The membrane has an initial deflection $f_{\text {in }}$.

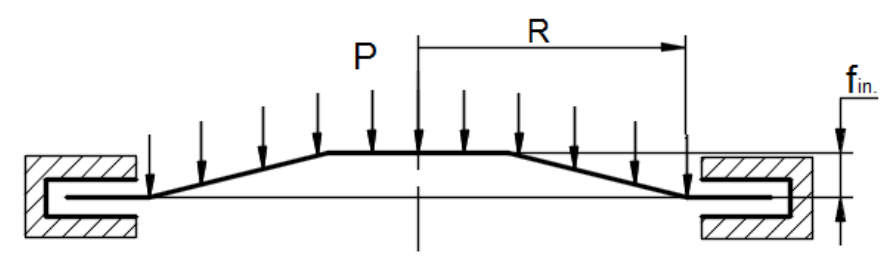

Figure 2. Position fixing diagram: membrane non-loaded state.

The measurement of membrane center additional deflection in the stability loss state followed such sequential steps.

1. After cooling the product, the capped jar was placed on the indicator device control plate that instrument being adjusted ready for measurements (see paragraph 1-3 below).

2. Using a sharp awl, a hole was pierced in the cap field (not on the membrane). The pressure in the container was compared with atmospheric pressure and the membrane did stepwise return to its original state of equilibrium. At that the indicator arrow deviated from the zero position. The instrumental readings were recorded.

3. Additional deflection of the membrane center in the stability loss state $f$, measured from the fixing contour plane, Figure 3, was found as the difference between the indicator reading and the membrane center initial deflection.

The membrane fixing contour possible deflection due to the action of vacuum in the container was neglected with respect to its insignificant value in comparison with the functional travel of the membrane center.

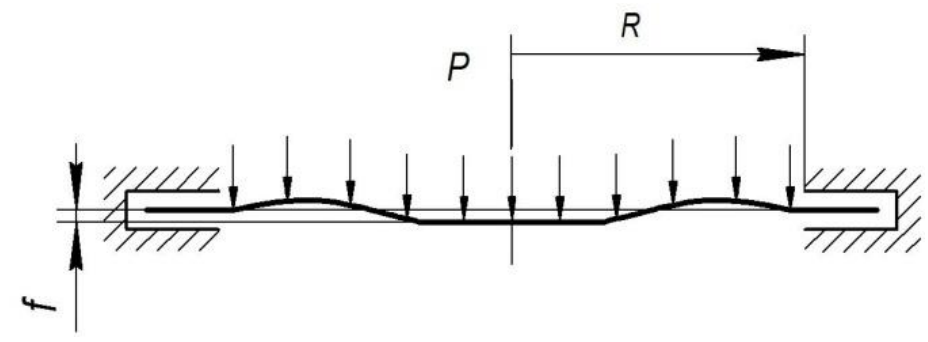

Figure 3. Position fixing diagram: membrane stability loss state 


\section{Description of methods and equipment}

Additional deflection and thickness of the membrane sheet were determined by instrumental methods on real glassware with a cap.

The deflection was measured using an indicator device Figure 4. The metering device consists of a tripod for measuring heads 1 , fixed on a horizontal control plate 2 bearing holes for arresters, a clock-type indicator 3, (measurement error $0,01 \mathrm{~mm}$ ), and two locking arresters 4 .

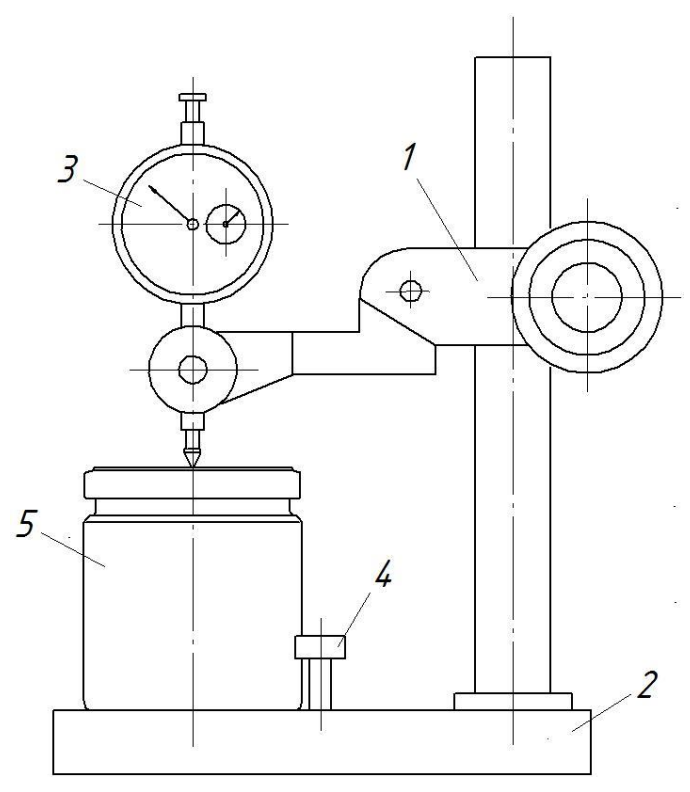

Figure 4. Indicator device.

1 - tripod; 2 control plate; 3 - dial gauge; 4 - locking stops; 5 - tested sample.

Preparing the device for measurements followed such sequential steps.

1. The tested sample placed its bottom on the control plate, the indicator measuring rod was raised thus the sample was wound up under the measuring head.

2. The indicator tip being brought into contact with the membrane flat section center after that the container position was fixed using two locking arresters, previously installed in the control plate holes suitable for the given container body dimensions.

3. The indicator clip terminal on the tripod released, the indicator measuring tip was adjusted to contact the cap membrane center so that the arrow on its small scale deviated from zero by an integer number of millimeters, but not less than the membrane's initial deflection twice value. Next the indicator was set to zero with fixing its position on a tripod.

Since the tested sample consists of several parts (jar, cap, sealing gasket of the cap) that have their own height tolerance, each sample will have its own height. Suffice to say is that only the glass containers height tolerance can vary between 1-3 mm. Therefore, for each tested sample measurement required is an individual presetting of the device according to procedure preparing the experimental sample.

The tin caps thickness was measured by a micrometer with an error of $0,01 \mathrm{~mm}$. 
The membrane equilibrium state kinds' mathematical simulation was performed using the plates and shells theory energy method described by numerous authors $[6,7,8]$. The membranes' operation in the mode of controlled stability loss is closely related to their material energy component, so it is most appropriate to use namely this method. According to the method above, the round plate total energy is represented as the sum of the zero-torque stress state energy, the bending energy, and the work of external pressure.

The membrane energy levels diagrams were built by mathematical modeling using computational methods in the Scilab environment $[14,15]$.

\section{Study of research results}

The experiment used a membrane with parameters $f_{\text {in }}=0,25 \mathrm{~mm}$; tin thickness $\delta=0.18$ $\mathrm{mm}$; membrane fixing contour radius $R=12 \mathrm{~mm}$, made of low-carbon steel. The measurements results showed that in the stability loss state, the membrane center additional deflection was $f=0,07 \mathrm{~mm}$, that is significantly less than the membrane center initial deflection $f_{\text {in }}=0,25 \mathrm{~mm}$, Figure 3 . That is, as a result of stability loss membrane working cone mirror deformation does not occur.

For a visual interpretation of the membrane operation, the membrane energy levels diagrams were built for its characteristic equilibrium states: loss of stability and load releasing. The energy levels diagrams allowed us to check the research results compliance with the main provisions of theoretical mechanics, in particular the Lagrange-Dirichlet theorem [16].

\section{Results and discussions}

\section{Derivation of the membrane system total energy scope equation.}

For calculating the system energy we use the initial equations from the plates and shells theory. The membrane system total energy is found similarly to the axi-symmetric bend energy for a circular plate [10]

$$
E_{t}=E_{m}+E_{b}-W
$$

where $E_{m}$ is the stress energy in the membrane middle surface,

$$
E_{m}=\frac{E \delta}{2} \int_{0}^{R}\left[\left(\frac{d^{2} \Phi}{d r^{2}}\right)^{2}+\left(\frac{1}{r} \frac{d \Phi}{d r}\right)^{2}-\frac{2 \mu}{r} \frac{d \Phi}{d r} \frac{d^{2} \Phi}{d r^{2}}\right] 2 \pi r d r
$$

$E_{b}$ is the bending energy,

$$
E_{b}=\frac{D}{2} \int_{0}^{R}\left[\left(\frac{d^{2} \omega}{d r^{2}}\right)^{2}+\left(\frac{1}{r} \frac{d \omega}{d r}\right)^{2}-\frac{2 \mu}{r} \frac{d \omega}{d r} \frac{d^{2} \omega}{d r^{2}}\right] 2 \pi r d r
$$

$W$ is the external load work,

$$
W=\int_{0}^{R} P \omega 2 \pi r d r=2 \pi P \int_{0}^{R} \omega r d r,
$$

where $E$ is the modulus of membrane material normal elasticity;

$\delta$ is the membrane (tinplate) thickness;

$R$ is the membrane fixing contour radius;

$\Phi$ is the stress function;

$r$ is the membrane current radius;

$\mu$ is the membrane material Poisson's ratio; 
$D=\frac{E \delta^{3}}{12\left(1-\mu^{2}\right)}$ is the cylindrical stiffness of the membrane;

$\omega$ is the membrane additional current deflection;

$P$ is the pressure (load) applied to the membrane.

Write an expression for $\frac{d \Phi}{d r}[10]$

$$
\frac{d \Phi}{d r}=\frac{E f^{2}}{6 R}\left(3 \frac{r}{R}-6 \frac{r^{3}}{R^{3}}+4 \frac{r^{5}}{R^{5}}-\frac{r^{7}}{R^{7}}\right),
$$

where $f$ is the membrane center additional deflection.

We introduce the dimensionless current radius $r_{1}=\frac{r}{R}$, then $d r_{1}=\frac{d r}{R} ; \frac{d \Phi}{d r}=\frac{1}{R} \frac{d \Phi}{d r_{1}}$; $\frac{d^{2} \Phi}{d r^{2}}=\frac{1}{R^{2}} \frac{d^{2} \Phi}{d r_{1}^{2}}$.

After substituting these references in (5), we get

$$
\frac{d \Phi}{d r_{1}}=\frac{E f^{2}}{6}\left(3 r_{1}-6 r_{1}^{3}+4 r_{1}^{5}-r_{1}^{7}\right) \text {. }
$$

Respectively

$$
\frac{d^{2} \Phi}{d r_{1}^{2}}=\frac{E f^{2}}{6}\left(3-18 r_{1}^{2}+20 r_{1}^{4}-7 r_{1}^{6}\right) .
$$

Entering the $R\left(r_{1}\right)=3 r_{1}-6 r_{1}^{3}+4 r_{1}^{5}-r_{1}^{7}$, we get (6) and (7) respectively in the form

$$
\frac{d \Phi}{d r_{1}}=\frac{E f^{2}}{6} R\left(r_{1}\right) \text { and } \frac{d^{2} \Phi}{d r_{1}^{2}}=\frac{E f^{2}}{6} \frac{d R}{d r_{1}} .
$$

Write (2) for the dimensionless current radius $r_{l}$, given that for $r=0 \rightarrow r_{l} \rightarrow 0$, and for $r=R \rightarrow r_{l}=1$

$$
E_{m}=\frac{2 \pi E \delta}{2} \int_{0}^{1}\left[\frac{1}{R^{4}}\left(\frac{d^{2} \Phi}{d r_{1}^{2}}\right)^{2}+\frac{1}{R^{4}}\left(\frac{1}{r_{1}} \frac{d \Phi}{d r_{1}}\right)^{2}-\frac{1}{R^{4}} \frac{2 \mu}{r_{1}} \frac{d \Phi}{d r_{1}} \frac{d^{2} \Phi}{d r_{1}^{2}}\right] R^{2} r_{1} d r_{1} .
$$

After transformations and substitutions of expressions (8), the equation (9) takes the form

$$
E_{m}=\frac{\pi E^{3} f^{4} \delta}{36 R^{2}} \int_{0}^{1}\left[r_{1}\left(\frac{d R}{d r_{1}}\right)^{2}+\frac{1}{r_{1}} R^{2}\left(r_{1}\right)-2 \mu R\left(r_{1}\right) \frac{d R}{d r_{1}}\right] d r_{1} .
$$

Denote the integral part of the equation (10)

$$
I_{1}=\int_{0}^{1}\left[r_{1}\left(\frac{d R}{d r_{1}}\right)^{2}+\frac{1}{r_{1}} R^{2}\left(r_{1}\right)-2 \mu R\left(r_{1}\right) \frac{d R}{d r_{1}}\right] d r_{1} .
$$

Then the equation of membrane middle surface stress energy is

$$
E_{m}=\frac{\pi E^{3} f^{4} \delta}{36 R^{2}} I_{1} .
$$

Now we consider the bending energy $E_{b}$ equation (3). 
In the case of plate elastic deformation, the approximate expression for additional deflections corresponds to the similar problem solution in the case of a small deflection plate [11],

$$
\omega=f\left(1-\frac{r^{2}}{R^{2}}\right)^{2}
$$

Write (12) for the dimensionless current radius $r_{l}$

$$
\begin{gathered}
\omega=f\left(1-r_{1}^{2}\right)^{2}=f\left(1-2 r_{1}^{2}+r_{1}^{4}\right), \\
\frac{d \omega}{d r_{1}}=4 f r_{1}\left(r_{1}^{2}-1\right) .
\end{gathered}
$$

Entering the $W_{1}\left(r_{1}\right)=r_{1}\left(r_{1}^{2}-1\right)$, we get (14) taking the form

$$
\frac{d \omega}{d r_{1}}=4 f W_{1}\left(r_{1}\right)
$$

respectively

$$
\frac{d^{2} \omega}{d r_{1}}=4 f \frac{d W_{1}}{d r_{1}}
$$

We write (3) for, the dimensionless current radius $r_{l}$, given that for $r=0 \rightarrow r_{l} \rightarrow 0$, and for $r=R \rightarrow r_{l}=1$

$$
E_{b}=\pi D \int_{0}^{1}\left[\frac{1}{R^{4}}\left(\frac{d^{2} \omega}{d r_{1}^{2}}\right)^{2}+\frac{1}{R^{4}}\left(\frac{1}{r_{1}} \frac{d \omega}{d r_{1}}\right)^{2}+\frac{1}{R^{4}} \frac{2 \mu}{r_{1}} \frac{d \omega}{d r_{1}} \frac{d^{2} \omega}{d r_{1}^{2}}\right] R^{2} r_{1} d r_{1} .
$$

After transformations and substitutions (15) and (16), the equation (17) takes the form

$$
E_{b}=\frac{16 f^{2} \pi D}{R^{2}} \int_{0}^{1}\left[r_{1}\left(\frac{d W_{1}}{d r_{1}}\right)^{2}+\frac{1}{r_{1}} W_{1}^{2}\left(r_{1}\right)+2 \mu W_{1}\left(r_{1}\right) \frac{d W_{1}}{d r_{1}}\right] d r_{1} .
$$

We denote the integral part of the equation (18)

$$
I_{2}=\int_{0}^{1}\left[r_{1}\left(\frac{d W_{1}}{d r_{1}}\right)^{2}+\frac{1}{r_{1 r}} W_{1}^{2}\left(r_{1}\right)+2 \mu W_{1}\left(r_{1}\right) \frac{d W_{1}}{d r_{1}}\right] d r_{1} .
$$

Then the bending energy equation is

$$
E_{b}=\frac{16 f^{2} \pi D}{R^{2}} I_{2}
$$

Now we consider the external load work $W$ equation (4).

We write (4) for, the dimensionless current radius $r_{l}$, given that for $r=0 \rightarrow r_{l} \rightarrow 0$, and for $r=R \rightarrow r_{l}=1$, so after substituting (13) and transformations

$$
W=2 \pi P f R^{2} \int_{0}^{1}\left(r_{1}-2 r_{1}^{3}+r_{1}^{5}\right) d r_{1} .
$$

Then, integrating performed, the external load work equation is

$$
W=\frac{\pi P f R^{2}}{3} \text {. }
$$

After substituting (11), (19) and (21) into the equation (1)

$$
E_{t}=\frac{\pi E^{3} f^{4} \delta}{36 R^{2}} I_{1}+\frac{16 f^{2} \pi D}{R^{2}} I_{2}-\frac{\pi P f R^{2}}{3} .
$$


We introduce a dimensionless additional deflection of the membrane center $\zeta=\frac{f}{\delta}$ and a dimensionless pressure (load) on the membrane $P^{*}=\frac{P R^{4}}{\delta^{4} E}$.

After substituting them in (22) and transformations, we get

$$
E_{t}=\frac{\pi}{R^{2}}\left[E \delta^{5} \frac{\zeta^{4}}{36} I_{1}+16 \delta^{2} D \zeta^{2} I_{2}-E \delta^{5} \frac{\zeta P^{*}}{3}\right] .
$$

Now we find integrals $I_{l}$ and $I_{2}$. Poisson's ratio for steel $\mu=0.35$.

Integrating $I_{l}$ we get

$$
I_{l}=48,8167+1,7047+1,4=51,9214 .
$$

Integrating $I_{2}$ we get

$$
I_{2}=0,5+0,1667+0=0,6667 .
$$

To graphically compare the membrane different equilibrium states energy levels we introduce the dimensionless total energy

$$
E_{t}^{*}=\frac{E_{n} R^{2}}{E \delta^{5}}
$$

After substituting (23) in (24) and calculation carried out, we get

$$
E_{t}^{*}=1,4423 \zeta^{4}+1,013 \zeta^{2}-0,3333 P^{*} \zeta .
$$

We reduce equation (25) to the equilibrium equation form. We differentiate equation (25) by $d \zeta$ and equate it to 0 . As a result, we get

$$
\zeta^{3}+0,3512 \zeta-0,0578 P^{*}=0
$$

\section{Analysis and verification of the obtained equilibrium equation correctness}

Substituting the experimentally obtained value of $f$ after stability loss in the equilibrium equation (26), we can obtain the corresponding critical pressure value.

If $f=0,07 \mathrm{~mm}$, the corresponding dimensionless deflection is $\zeta=0,39$. Expressing from (26) $P^{*}$ we get

$$
P^{*}=\frac{\zeta^{3}+0,3512 \zeta}{0,0578},
$$

where, for the case under consideration, we get $P_{1}^{*}=3,3958$. Accordingly we find $P_{1}$ from

$$
P=\frac{P^{*} \delta^{4} E}{R^{4}}
$$

where from, for the case under consideration, we get $P_{l}=0.0326 \cdot 10^{6} \mathrm{~Pa}$.

The calculated value of $P_{l}$ is close to the value of cap membranes pressure stability loss $P_{l}=0,03 \mathrm{MPa}$, specified by the jar caps manufacturer [17]. The calculated pressure value $P_{1}$ deviation from the experimental one may be due to the hardness class of the tin plate used to produce the membrane-equipped cap.

After the stability loss, the membrane shifts to the stable equilibrium state. The measurement results showed that in the stability loss state, the additional deflection of the membrane center $f=0,07 \mathrm{~mm}$, significantly less than the initial deflection of the membrane center $f_{\text {in }}=0,25 \mathrm{~mm}$, Figure 3 . That is, as a result of stability loss membrane working cone mirror deformation does not occur. 
These additional deflection parameters guarantee the membrane's performance over a wide range of drop between the system's back pressure (in the autoclave) and the container's internal pressure throughout the entire product heat treatment process. As $P_{l}$ hazardous value usually considered is this one greater than 0,07 MPa. Then during sterilization (thermal exposure or cooling start stages), there may be a malfunction of the control button due to the metal elastic deformation transition into plastic one.

The degree of tin hardness is increased to improve its rigidity. Through hardness increasing compensated is the decrease in tin strength which inevitably occurs in the case of the rolled steel thickness decrease. In the range of tin thickness used for caps, the range of hardness changes is constant and narrow.

\section{Analysis and explanation of energy levels at membrane equilibrium states}

We shall consider the energy levels of different membrane equilibrium states under two different loads that correspond to critical pressures: stability loss $P_{1}^{*}$ and load release $P_{2}^{*}$. They are shown in Figure 5, and are built using the graphically interpreted equation (26) for pressures $P_{1}^{*}$ and $P_{2}^{*}$. At values $P_{1}^{*}=3,12$ and $P_{2}^{*}=0,52$, corresponding to $P_{l}=0,03 \cdot 10^{6} \mathrm{~Pa}$ and $P_{2}=0.005 \cdot 10^{6} \mathrm{~Pa}$, for the membrane center initial dimensionless deflection $\zeta_{\text {in }}=1,39$, which is defined as $\zeta_{\text {in }}=\frac{f_{\text {in }}}{\delta}$, and the membrane parameters given above. Other design parameters $\mu=0,35 ; E=190 \cdot 10^{9} \mathrm{~Pa}$. Here $E$ and $\mu$ correspond to low-carbon steel.

The diagrams show that there is a minimum energy value for both pressures. The pressure $P_{1 \min }^{*}$ corresponds to the moment when it causes the center's certain additional deflection, which reached the membrane abruptly loses stability, passing into another, opposite, state of equilibrium (Figure 3). This state corresponds to the center's additional deflection which value was measured using an indicator device.

The pressure $P_{2 \min }^{*}$ corresponds to the moment when the membrane, being unloaded, abruptly resumes its shape under its internal energy action, but it does not yet reach the initial state of equilibrium, since $P_{2 \min }^{*} \neq 0$.

This type of energy criterion curves for critical pressures fully corresponds to the wellknown Lagrange-Dirichlet theorem [16], according to which the main stable equilibrium state is the plate state corresponding to the pressure $P_{1}^{*}$, since the pressure $P_{1 \text { min }}^{*}$ corresponds to the energy level, minimum relative to other adjacent states.

The membrane initial state when no external load applied, i.e. $P^{*}=0$, and it is in a state of equilibrium, is taken as zero energy value level. This state is the same for both pressures, since after load full release, the membrane returns to the initial state of equilibrium when the controlled stability loss mode maintained. 


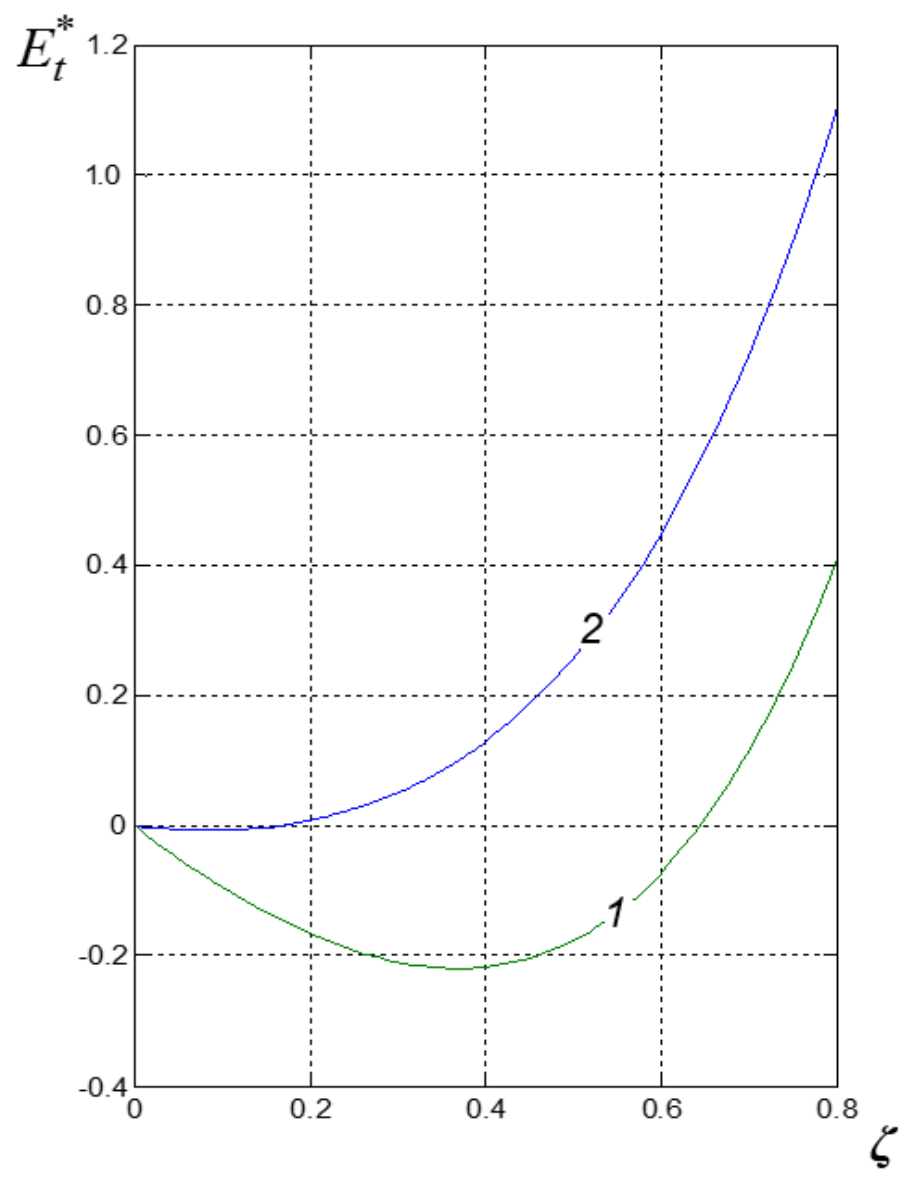

Figure 5. Diagrams of the membrane energy levels:

1 - for the stability loss pressure $P_{1}^{*} ; \quad 2$ - for the load release pressure $P_{2}^{*}$.

\section{Conclusions}

1. The equation (26), obtained by solving the local energy effects equations, is a scope equation that characterizes and represents the energy transformations in the membrane body.

2. It is experimentally found that the membrane additional deflection when operational state is significantly less than its initial deflection.

3. The quoted calculation demonstrates the resulting scope equation's adequacy to the initially bent real metal plates in case of load counteracting the initial deflection, in particular for glass canning containers' metal caps membranes

4. The membrane energy levels diagrams obtained using equation (26) for characteristic equilibrium states are correct and agree with the Lagrange-Dirichlet theorem. Therefore, the equation (26) can be used in the industry manufacturing the canned food products metal packaging materials, as well as in the instrument building industry.

5. The round elastic plate controlled stability loss computational mode has been build, checked and developed. 


\section{References}

1. Vatrenko O. (2014), Membrany kryshok konservnoi sklianoi tary, Upakovka, 6, pp. 26-29.

2. Schiester S. (2018), A new twist, Compact steel, 01, p. 22.

3. Ugural A. (2018), Plates and shells: theory and analysis, fourth edition, CRC Press, Taylor \& Fransis group, London, New York.

4. Szilard R. (2004), Theories and application of plate analysis: Classical, Numerical and Engineering Methods, John Wiley \& Sons, Inc. Hoboken.

5. Aleksandrov A., Potapov V. (1990), Osnovy teorii uprugosti i plastichnosti, Vysshaia shkola, Moscow.

6. Reddy J. (2007), Theory and analysis of elastic plates and shells, second edition, CRC Press, Taylor \& Fransis group, London, New York.

7. Ventsel E., Krauthammer T. (2001), Thin plates and shells: Theory, analysis and applications, Marcel Dekker, Inc., New York, Basel.

8. Ugural A., Fenster S. (2012), Advanced Mechanics of Materials and Applied Elasticity, 5th ed. Prentice-hall, Upper Saddle River, NJ.

9. Bradford M., Roufegarinejad A., Vrcelj Z. (2006), Elastic buckling of thin-walled circular tubes containing an elastic infill, International Journal of Structural Stability and Dynamics, 6(4), pp. 457-474.

10. Volmir A. (1956), Gibkie plastinki i obolochki, Izdatelstvo tekhniko-teoreticheskoi literatury, Moscow.

11. Timoshenko S., Voinovskii-Kriger S. (1966), Plastinki i obolochki, Nauka, Moskva.

12. Frank Pai P. (April 2007) Total-Lagrangian Formulation and Finite-Element Analysis of Highly Flexible Plates and Shells. Mathematics and Mechanics of Socaps, 12(2), pp. 213-250.

13. Vatrenko O. (2015), Modeliuvannia roboty membran vakuumnykh kryshok: progyn, tovshchyna, Naukovi pratsi ONAKT, 48, pp. 150-154.

14. Eros B. Vieira, Wesley F. Busch, Lizandro S. Santos (2018), Application of Scilab/Xcos for process control applied to chemical engineering educational projects, Computer Applications in Engineering Education, 27(1), pp. 154-165.

15. Borutzky W. (2009), Bond Graph Methodology, Springer, London.

16. Ishlinskii L. (1985), Mekhanika. Idei, zadachi, prilozheniia, Vysshaia shkola, Moscow.

17. (2006), Rukovodstvo po obrabotke kryshek Twist-off, Silgan White Cap, Hannover. 